\title{
Cloud-resolving model simulations with one- and two-way couplings via the weak temperature gradient approximation
}

Article

Published Version

Daleu, C. L., Woolnough, S. J. and Plant, R. S. (2012) Cloudresolving model simulations with one- and two-way couplings via the weak temperature gradient approximation. Journal of the Atmospheric Sciences, 69 (12). pp. 3683-3699. ISSN 1520-0469 doi: https://doi.org/10.1175/JAS-D-12-058.1 Available at https://centaur.reading.ac.uk/28698/

It is advisable to refer to the publisher's version if you intend to cite from the work. See Guidance on citing.

To link to this article DOI: http://dx.doi.org/10.1175/JAS-D-12-058.1

Publisher: American Meteorological Society

All outputs in CentAUR are protected by Intellectual Property Rights law, including copyright law. Copyright and IPR is retained by the creators or other copyright holders. Terms and conditions for use of this material are defined in the End User Agreement.

www.reading.ac.uk/centaur 
Central Archive at the University of Reading

Reading's research outputs online 


\title{
Cloud-Resolving Model Simulations with One- and Two-Way Couplings via the Weak Temperature Gradient Approximation
}

\author{
C. L. Daleu, S. J. Woolnough, and R. S. Plant \\ Department of Meteorology, University of Reading, Reading, United Kingdom
}

(Manuscript received 22 February 2012, in final form 22 May 2012)

\begin{abstract}
A cloud-resolving model is modified to implement the weak temperature gradient approximation in order to simulate the interactions between tropical convection and the large-scale tropical circulation. The instantaneous domain-mean potential temperature is relaxed toward a reference profile obtained from a radiative-convective equilibrium simulation of the cloud-resolving model. For homogeneous surface conditions, the model state at equilibrium is a large-scale circulation with its descending branch in the simulated column. This is similar to the equilibrium state found in some other studies, but not all. For this model, the development of such a circulation is insensitive to the relaxation profile and the initial conditions.

Two columns of the cloud-resolving model are fully coupled by relaxing the instantaneous domain-mean potential temperature in both columns toward each other. This configuration is energetically closed in contrast to the reference-column configuration. No mean large-scale circulation develops over homogeneous surface conditions, regardless of the relative area of the two columns. The sensitivity to nonuniform surface conditions is similar to that obtained in the reference-column configuration if the two simulated columns have very different areas, but it is markedly weaker for columns of comparable area. The weaker sensitivity can be understood as being a consequence of a formulation for which the energy budget is closed. The referencecolumn configuration has been used to study the convection in a local region under the influence of a largescale circulation. The extension to a two-column configuration is proposed as a methodology for studying the influence on local convection of changes in remote convection.
\end{abstract}

\section{Introduction}

A key issue in understanding tropical climate and its variability is our limited knowledge about the interactions between moist convection and the large-scale tropical circulation. General circulation models (GCMs) are a powerful tool for studying the large-scale circulation, but they suffer from a range of problems associated with the need to parameterize convection (e.g., Lin et al. 2006; Dai 2006; Lin et al. 2008; Randall et al. 2007). Cloud-resolving models (CRMs) are a powerful tool for studying moist convection but, as normally used, the large-scale circulation is prescribed (e.g., Robe and Emanuel 1996; Tompkins 2001; Xu et al. 2002; Petch et al. 2006). Because of the large range of scales involved, there are relatively few studies that explicitly simulate both moist convection and the large-scale tropical circulation (e.g., Grabowski

Corresponding author address: C. L. Daleu, Department of Meteorology, University of Reading, Earley Gate, P.O. Box 243, Reading RG6 6BB, United Kingdom.

E-mail: c.l.daleu@pgr.reading.ac.uk et al. 2000; Shutts and Palmer 2007; Liu et al. 2009; Holloway et al. 2012).

The weak temperature gradient (WTG) approximation (e.g., Sobel and Bretherton 2000) has been exploited in recent years as a possible basis for modeling the interactions between convection and the large-scale circulation. An alternative approach, based on the coupling to two-dimensional gravity wave modes, is discussed by Kuang $(2008,2011)$. Various WTG studies have coupled a CRM simulation to a reference reservoir column through a parameterization of the large-scale circulation (e.g., Raymond and Zeng 2005; Sobel et al. 2007; Sessions et al. 2010; Wang and Sobel 2011). The coupling to a reservoir may be justified by assuming that the region to be simulated is small in comparison with its surroundings. A typical situation would be to consider that surface conditions [e.g., high sea surface temperature (SST)] in a localized region are different from the surroundings. It is expected that an ascending (or descending) branch arises over the local region, with compensating motion within the surroundings that is negligibly weak because of the differences in areas. A typical objective is to 
study the effects of coupling to the large area on the precipitation within the explicitly simulated local area.

We present here a system in which two regions are explicitly simulated, the regions being coupled through a large-scale circulation derived from the WTG approximation. Such a system provides an expanded framework that could be used to study a wider range of problems, including cases where the ascending and descending branches of the circulation occupy comparable areas. For example, transitions between shallow and deep convection could be studied for circumstances in which these are locally or remotely forced. The two regions may be of arbitrary relative area; although in the results to be shown, we will focus on cases with either equal area or where the areas are very different. For the situation of equal areas, one would expect to find the largest differences from the reference-column approach. An important question to ask, therefore, is whether those differences are actually substantial, or whether a reference-column approach might have been sufficient. For the situation with very different areas, one might expect to find similar behavior using either a referencecolumn or a two-column approach. We shall show that this expectation is indeed borne out by results for various configurations, but also that under fully homogeneous conditions there are some differences between the approaches that remain.

Many cloud-resolving simulations of deep convection have been performed for radiative-convective equilibrium (RCE) (e.g., Robe and Emanuel 1996; Tompkins and Craig 1998a; Cohen and Craig 2006). In such a configuration, the simulation is run for a sufficiently long time until the heating induced by convection balances the (often prescribed) cooling due to radiation. Other popular CRM configurations include idealized studies with predefined advective large-scale forcing, possibly based on experimental campaigns (e.g., Vincent 1994; Tompkins 2001; Xu et al. 2002; Petch et al. 2006). The processes occurring within the simulated system are assumed to be fast compared to the time scale characterizing changes in the large-scale flow. Therefore, CRM simulations usually disconnect convective development from any evolution of a large-scale environmental flow that is fully externalized. It must be recognized that these, and many other such studies, have undoubtedly provided much useful insight. In practice, however, there may be close links between tropical convection and the largescale flow, such that treating one as the forcing for the other is not necessarily appropriate, particularly in nonequilibrium conditions (Mapes 1997; Holloway and Neelin 2010; Masunaga 2012).

To understand how convection influences the largescale circulation and how the large-scale circulation in turn influences convective development, there is a need for approaches in which both the large-scale and the convective elements are simulated. This is becoming possible in large-domain, high-resolution experiments, such as those conducted at the Earth Simulator Center (Liu et al. 2009) and in projects such as Cascade (e.g., Holloway et al. 2012). Another alternative approach is superparameterization (Randall et al. 2003), in which the subgrid flow within each grid square of a GCM is determined from an embedded CRM. An alternative was proposed by Kuang et al. (2005), who reduce the scale difference between convection and large-scale circulation using a diabatic acceleration and rescaling approach in order to be able to resolve both scales within a three-dimensional CRM. For too large a rescaling, the troposphere becomes deeper and the length of convective life cycle is increased relative to synoptic phenomena (Garner et al. 2007). A common disadvantage of all of these approaches is that integrations remain computationally expensive, so that it is not practical to study large sets of multiple experiments to test mechanisms and assess sensitivities.

The WTG approximation of Sobel and Bretherton (2000) has proved to be a useful and computationally cheap framework to diagnose a large-scale flow and allow its interaction with convection in a limited-area model representing a region of the atmosphere near the equator. The underlying physical idea behind the approximation is that the role of gravity waves over a large area of the tropics is to redistribute density anomalies produced by surface fluxes or radiation and hence to maintain near-uniform density on isobaric surfaces (Bretherton and Smolarkiewcz 1989; Mapes and Houze 1995; Yano and Bonazzola 2009). It can be applied only near the equator, where the action of the Coriolis force is almost zero. In that case, horizontal gradients and time tendencies of potential temperature are both negligible compared to its vertical gradients. A large-scale vertical velocity can then be derived as that which is sufficient to balance the simulated heating and accordingly maintain the horizontal domain-mean potential temperature.

Sobel and Bretherton (2000) implemented the WTG approximation to a single-column model (SCM) version of the first quasi-equilibrium tropical model (QTCM1) of Neelin and Zeng (2000). For unchanged surface conditions, the climatological mean rain rate obtained was not significantly different from that in the corresponding SCM version of the QTCM1 without the WTG parameterization of a large-scale circulation. For the case of inhomogeneous surface conditions, Sobel et al. (2007) and Bellon and Sobel (2010) used the SCM version of the fifth-generation Goddard Earth Observing 
System (GEOS-5) GCM and the second version of the QTCM (QTCM2; Sobel and Neelin 2006), respectively, to explain the existence of two distinct quasi-steady states.

Raymond and Zeng (2005) used the WTG approximation to diagnose a large-scale circulation for use with CRM simulations. In their case, the domain-mean largescale vertical velocity was derived from the requirement that the instantaneous domain-mean potential temperature profile be maintained close to a reference profile, the reference state itself having previously been determined from the RCE configuration of the same model at a given SST. In a homogeneous environment (i.e., with the same surface conditions as for the RCE configuration), a large-scale circulation develops with descent in the simulated region. Analogous simulations were performed for inhomogeneous surface conditions by Sessions et al. (2010) and showed that two distinct equilibrium states could arise in the CRM when coupled to a fixed reference state through the WTG parameterization of large-scale circulation.

In WTG simulations that use a reference column, the heat and moisture budgets of the system are, by design, not closed. For example, suppose that large-scale descent is diagnosed within the simulated region by mass continuity that should be accompanied by ascent in a neighboring region. The ascent will act to cool and moisten the neighboring region. Such feedback effects are omitted in the reference-column approach, since they can be assumed to be weak if distributed over a very large area. This paper describes how a WTG-derived circulation can be implemented in a configuration that explicitly incorporates such feedbacks and thereby allows one to remove the assumption of a large difference in areas. This can be achieved by coupling two columns of the atmosphere, each of which is simulated by a CRM. The profiles in the columns are not specified a priori but rather are computed during the simulation, which allows a full two-way coupling between the columns. Although some previous investigations have been performed that coupled together two columns of an SCM (e.g., Nilsson and Emanuel 1999; Raymond and Zeng 2000; Shaevitz and Sobel 2004), in this paper CRM simulations are used to represent clouds in the two columns. The formulation and behavior of the coupled two-column system is presented, and, where appropriate, is compared with the behavior of analogous reference-column experiments.

The paper is organized as follows. Section 2 describes the CRM used and presents its RCE state, which will be used later to provide a suitable reference state. Section 3 details our implementation of the WTG approximation in order to diagnose a large-scale circulation, which is then applied within CRM simulations that use either a reference column (section 4a) or else two coupled CRM columns (section 4b). The simulations compared in section 4 are for homogeneous surface conditions. In section 5 we compare reference columns and coupled columns for inhomogeneous surface conditions. Conclusions are presented and the implications of our study are discussed in section 6 .

\section{Model description and radiative-convective simulations}

\section{a. Model description}

The CRM used in this study is based on the Met Office large-eddy model (LEM) (Shutts and Gray 1994; Petch and Gray 2001). It has a five-category prognostic microphysical scheme (Swann 1998; Brown and Heymsfield 2001) with prognostic variables for the mixing ratios of cloud water, rain, ice, graupel, and snow, and for the number concentrations of ice, graupel, and snow. The model has been used for a wide range of case study simulations, including shallow convection, deep convection, and the transitions between them (e.g., Abel and Shipway 2007; Petch and Gray 2001; Woolnough et al. 2010).

Here, the model is used in a two-dimensional configuration, with a length of $128 \mathrm{~km}$ and a height of $20 \mathrm{~km}$. The domain is large enough to accommodate an ensemble of convective clouds together with the compensating subsidence motions. At the same time, it is not so large as to allow spontaneous mesoscale organization of convection (Tompkins 2000; Bretherton and Smolarkiewcz 1989). There are 60 levels in the vertical, on a stretched grid with finer resolution closer to the surface. The midtropospheric grid spacing is about $330 \mathrm{~m}$, and the horizontal grid spacing is $500 \mathrm{~m}$. The lateral boundary conditions are periodic for all prognostic variables. The lower boundary is an ocean surface, with a roughness length of $2 \times 10^{-4} \mathrm{~m}$ and a prescribed spatially uniform and time-independent SST. The top and bottom of the domain are rigid lids. A Newtonian damping layer is used aloft (from 15 to $20 \mathrm{~km}$ ) in order to prevent vertically propagating gravity waves from being reflected back into the domain.

Aside from any large-scale circulation that might develop, the forcing for a CRM simulation consists of a horizontally homogeneous and noninteractive cooling through the troposphere. We will refer to such cooling as radiative cooling. It has the value of $1.5 \mathrm{~K} \mathrm{day}^{-1}$ up to $12 \mathrm{~km}$ and then decreases linearly with pressure to reach the value of $0 \mathrm{~K} \mathrm{day}^{-1}$ at about $15 \mathrm{~km}$. In the alongdomain direction, the domain-mean wind is relaxed toward zero with a relaxation time scale of $2 \mathrm{~h}$. The 

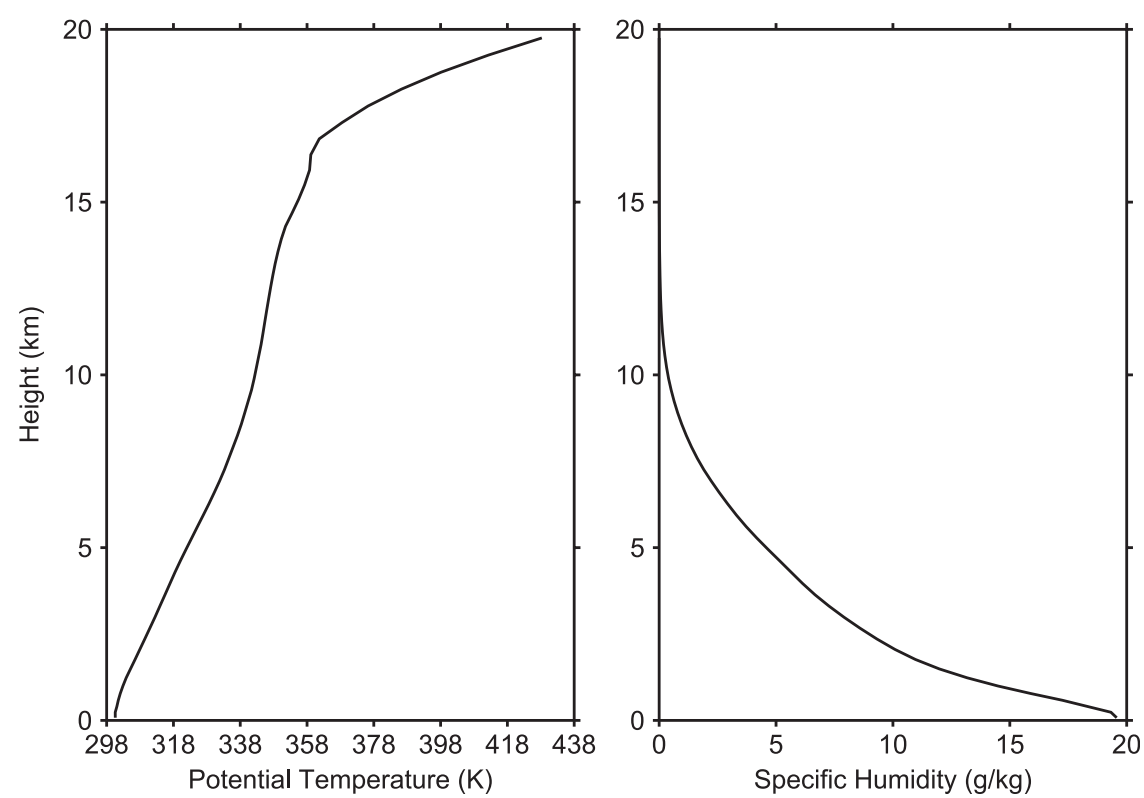

FIG. 1. (left) Potential temperature and (right) specific humidity profiles for the RCE state of the control integration with an SST of $302.7 \mathrm{~K}$.

relaxation is applied to prevent the development of along-domain wind shear that may otherwise occur in two-dimensional simulations (Held et al. 1993; Tompkins 2000; Mapes and Wu 2001) and that would encourage the formation of squall lines (e.g., Rotunno et al. 1988; Robe and Emanuel 2001; Tao et al. 1999). In the across-domain direction, a wind of $5 \mathrm{~m} \mathrm{~s}^{-1}$ is imposed, a choice that affects the simulations via the bulk aerodynamic formulas that are used to compute surface fluxes.

\section{b. Radiative-convective equilibrium simulations}

Several radiative-convective equilibrium simulations are performed for different SST. In each case the model is initialized with temperature and humidity profiles measured during the Tropical Ocean and Global Atmosphere Coupled Ocean-Atmosphere Response Experiment (TOGA COARE) from the western Pacific warm pool (Webster and Lukas 1992). The model is run for 40 days, and averages over the last 20 days are used to define the model state and statistics at equilibrium. The control integration has an SST of $302.7 \mathrm{~K}$, corresponding to the mean value of SST measured during the TOGA COARE field campaign. The mean rain rate obtained for the last 20 days is $4.77 \mathrm{~mm} \mathrm{day}^{-1}$, compared to $4.80 \mathrm{~mm}$ day $^{-1}$ for the surface evaporation rate. Figure 1 shows the potential temperature and specific humidity profiles at RCE for the control integration. These profiles are used to define the referencecolumn profiles in the WTG experiments described in section $4 \mathrm{a}$, as well as providing initial conditions for some of the experiments.
Figure 2 shows the deviation from the control profiles for a set of additional experiments with SSTs of 301.7, $303.7,304.7$, and $305.7 \mathrm{~K}$. These simulations are used to provide a set of additional initial profiles for the WTG simulations.

\section{Implementation of the WTG approach}

In flux form the equation for the evolution of potential temperature is

$$
\frac{\partial \theta}{\partial t}+\nabla_{h} \cdot(\mathbf{v} \theta)+\frac{1}{\rho} \frac{\partial}{\partial z}(\rho \omega \theta)=S_{\theta}
$$

and the continuity equation is

$$
\nabla_{h} \cdot \mathbf{v}+\frac{1}{\rho} \frac{\partial}{\partial z}(\rho \omega)=0,
$$

where $\rho$ is a horizontally uniform reference density, $\mathbf{v}$ is the horizontal velocity vector, $\omega$ is the vertical velocity, $\nabla_{h}$ is the horizontal gradient operator, and $S_{\theta}$ is the diabatic source term.

In the tropical free troposphere, large-scale horizontal gradients and time tendencies of temperature are small because buoyancy anomalies are rapidly redistributed by gravity waves. Thus, the first-order balance in Eq. (1) is that vertical advection of potential temperature acts to (nearly) balance the diabatic heating; that is,

$$
\bar{\omega} \frac{\partial \bar{\theta}}{\partial z} \approx \bar{S}_{\theta},
$$



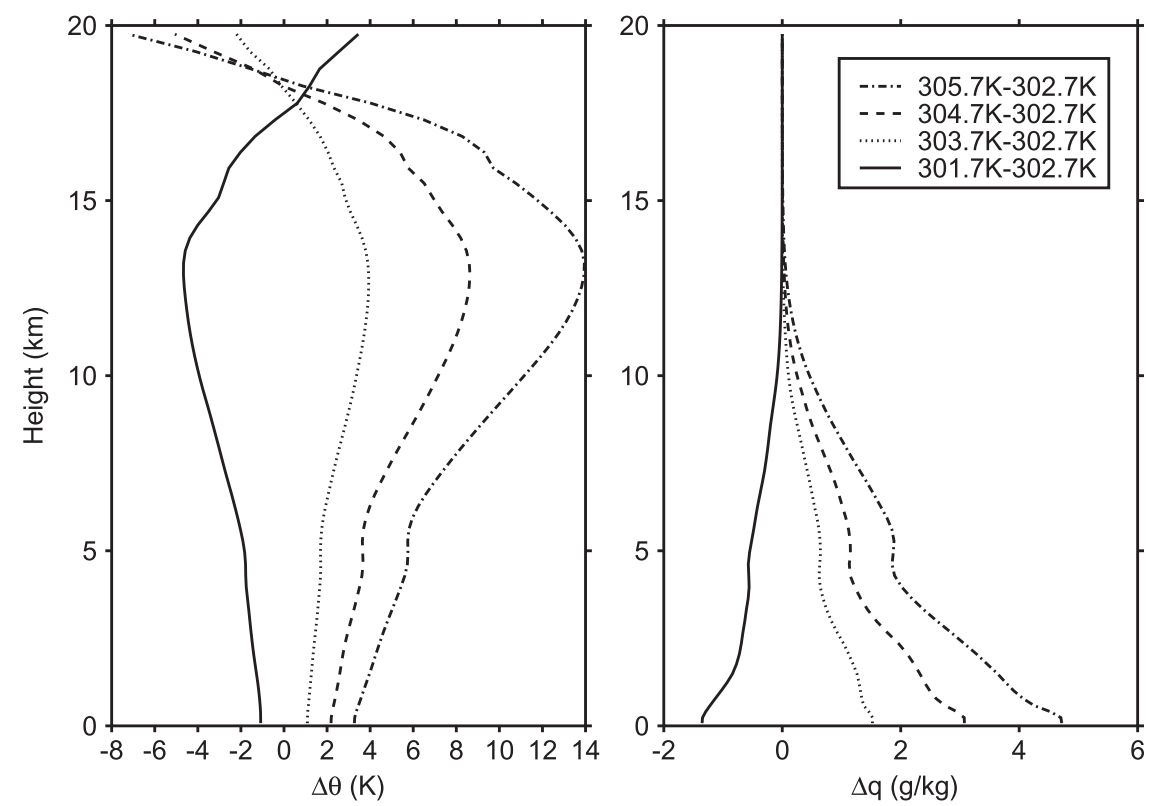

FIG. 2. Deviations from the control integration for RCE profiles of (left) potential temperature and (right) specific humidity for the RCE simulations with SSTs of 301.7, 303.7,304.7, and $305.7 \mathrm{~K}$.

where the overbar represents a suitably filtered largescale flow.

The two-column formulation considered in this study is illustrated by Fig. 3. The subscripts 1 and 2 are used to label the horizontal domain-mean properties in each column; $L$ is the length of the full system to be represented (i.e., of the two columns), which need not necessarily correspond to the domain size of the CRM, for reasons to be explained below. The individual column lengths are $L_{1}=(1-\varepsilon) L$ and $L_{2}=\varepsilon L$, so that $\varepsilon=L_{2} /$ $\left(L_{1}+L_{2}\right)$. The generalization to a three-dimensional implementation is entirely straightforward.

Sobel and Bretherton (2000) applied Eq. (3) in order to diagnose the large-scale vertical velocity $\bar{\omega}$ that would be necessary to eliminate the horizontal potential temperature gradient between a single simulated column and a reference state. In the present study, we apply a weaker constraint that the large-scale circulation acts to reduce the difference in the column-mean potential temperature profiles between the two simulated columns over a specified time scale. Thus,

$$
\bar{\omega}_{2} \frac{\partial \bar{\theta}_{2}}{\partial z}-\bar{\omega}_{1} \frac{\partial \bar{\theta}_{1}}{\partial z}=\frac{f(z)}{\tau}\left(\bar{\theta}_{2}-\bar{\theta}_{1}\right)
$$

The relaxation form is similar to that applied by Raymond and Zeng (2005), Sessions et al. (2010), and others. The dimensionless function $f(z)$ is introduced to allow the WTG adjustment rate $\lambda(z)=f(z) / \tau$ to be a function of height. Choices of the vertical functions used in this study are presented and motivated in section $3 \mathrm{a}$. The parameter $\tau$ is the time scale over which the largescale circulation acts to balance the heating associated with the simulated convection: it can be thought of as being related to the time for gravity waves to propagate across the system and so is related to the system size. Although the relative size of the columns $\varepsilon$ does enter into the diagnosis of the large-scale circulation below, the total system size plays no role except in the sense just described; that is, the system size that one has in mind will influence the choice of a suitable value for $\tau$. An

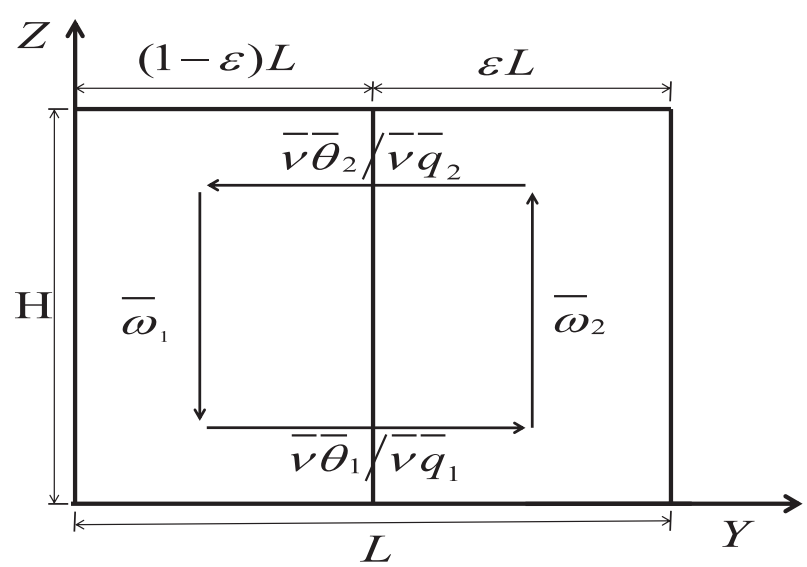

FIG. 3. Schematic of the two-column model. 
important implication is that the size of the simulation domains can be chosen for practical convenience and need not correspond with the implicit size of the physical system.

Equation (4) contains two unknowns, $\bar{\omega}_{1}$ and $\bar{\omega}_{2}$. By applying the two-column form of the mass continuity equation,

$$
(1-\varepsilon) \bar{\omega}_{1}+\varepsilon \bar{\omega}_{2}=0
$$

the WTG-derived vertical velocity $\bar{\omega}_{1}$ can be calculated as

$$
\bar{\omega}_{1}=\frac{f(z)}{\tau} \frac{\bar{\theta}_{1}-\bar{\theta}_{2}}{\left(\partial \bar{\theta}_{1} / \partial z\right)+[(1-\varepsilon) / \varepsilon]\left(\partial \bar{\theta}_{2} / \partial z\right)},
$$

with $\bar{\omega}_{2}$ then easily obtained from Eq. (5). It may be noticed that a reference-column formulation can be recovered in the strict limit of $\varepsilon=0$ (or $\varepsilon=1$ ), in which case the WTG-derived velocity vanishes in the infinitely large reference column, and agrees with that used by other studies for the negligibly small column, specifically

$$
\bar{\omega}=\frac{f(z)}{\tau}\left(\frac{\bar{\theta}-\bar{\theta}_{\mathrm{ref}}}{\partial \bar{\theta} / \partial z}\right) .
$$

Assuming that the stratification remains similar in both columns for $0<\varepsilon<1$, the two columns are relaxed toward each other with effective relaxation time scales that are different for the two columns, and longer than in the corresponding reference-column limit, being rescaled by factors of approximately $1 / \varepsilon$ and $1 /(1-\varepsilon)$ for columns 1 and 2, respectively.

The WTG approximation only holds if the horizontal gradient in potential temperature is much smaller than the vertical gradient. In this two-column formulation, the requirement is that $\left(\left|\bar{\theta}_{1}-\bar{\theta}_{2}\right| / L\right) \ll\left[\left(\partial \bar{\theta}_{1} / \partial z\right)+\right.$ $\left.\left(\partial \bar{\theta}_{2} / \partial z\right)\right]$. However, in the model simulations, the prescribed radiative cooling profile may sometimes result in a very weak static stability in the upper troposphere that would in turn cause the WTG calculation to produce very large and unphysical large-scale vertical velocities. To prevent such large vertical velocities from developing in the simulations, a lower bound of $1 \mathrm{~K} \mathrm{~km}^{-1}$ is imposed on the static stability when using Eq. (6) to calculate the WTG velocity. A similar condition was imposed in Raymond and Zeng (2005). The impact of the limiter is to reduce the strength of the diagnosed WTG circulation and hence the induced temperature tendencies.

Velocities derived from Eqs. (5) and (6) are named the WTG vertical velocities and are denoted as $\bar{\omega}_{1}^{\text {WTG }}$ and $\bar{\omega}_{2}^{\mathrm{WTG}}$, respectively. They are used to compute the large-scale advective tendencies of heat and moisture in the two columns. The change in the intensive, horizontalcolumn mean quantity $\bar{\chi}$ due to the WTG-derived largescale circulation is written in flux form as

$$
\frac{\partial \bar{\chi}}{\partial t}+\nabla_{h} \cdot\left(\mathbf{v}^{\mathrm{WTG}_{\bar{\chi}}}\right)+\frac{1}{\bar{\rho}} \frac{\partial}{\partial z}\left(\overline{\rho \omega}^{\mathrm{WTG}} \overline{\bar{\chi}}\right)=0 .
$$

By assuming that the horizontal flow $\mathbf{v}^{\text {WTG }}$ at each level is either all into or all out of each column and using an upwind advection scheme, the tendency of $\bar{\chi}_{1}$ due to the WTG circulation can written as

$\left(\frac{\partial \bar{\chi}_{1}}{\partial t}\right)_{\mathrm{WTG}}=\frac{\chi_{*}}{\bar{\rho}} \frac{\partial}{\partial z}\left(\overline{\rho \omega}_{1}^{\mathrm{WTG}}\right)-\frac{1}{\bar{\rho}} \frac{\partial}{\partial z}\left(\overline{\rho \omega}_{1}^{\mathrm{WTG}} \bar{\chi}_{1}\right)$

and similarly for $\bar{\chi}_{2}$. Here $\chi_{*}$ is the appropriate upwind value of $\bar{\chi}$, and the vertical derivative is calculated using an upwind scheme. Equation (9) is used to calculate WTG advective tendencies of potential temperature and water vapor only. The WTG circulation does not advect any form of hydrometeor.

The WTG calculations are performed on a coupling time step $d t_{\mathrm{WTG}}$ of $10 \mathrm{~min}$. The choice of $d t_{\mathrm{WTG}}$ is essentially arbitrary and might be thought of as equivalent to the time step of a general circulation model. A sensitivity study of the results to $d t_{\mathrm{WTG}}$ was done for values ranging between 0.5 and $30 \mathrm{~min}$. In most respects the nature of the results was unchanged and, in particular, no significant variations in the equilibrium mean rain rates were found.

\section{a. WTG adjustment profiles}

For most of our experiments, we use a WTG time scale that is constant with height in the free troposphere, that is,

$$
f(z)=f_{1}(z)=1 \text { for } z_{\mathrm{BL}}<z \leq z_{\mathrm{top}},
$$

where $z_{\text {top }}$ is the top of the domain. In the boundary layer, the WTG scaling approximation of Eq. (3) does not hold. We follow the approach of Sobel and Bretherton (2000) by not using the WTG approximation to calculate the large-scale vertical velocity in the boundary layer but rather linearly interpolate in height from the diagnosed WTG vertical velocity at a level $z_{\mathrm{BL}}$ a little above the boundary layer to a prescribed value of zero at the surface. Here we choose $z_{\mathrm{BL}}$ to be a model level at about $1.5 \mathrm{~km}$.

In some sensitivity experiments, we use a WTG adjustment rate profile given by 
$f(z)=f_{2}(z)=\left\{\begin{array}{lll}\sin \left(\frac{\pi z}{z_{t}}\right) & \text { for } & z_{\mathrm{BL}} \leq z \leq z_{t} \\ 0 & \text { for } & z>z_{t}\end{array}\right.$

to test the sensitivity of the results to the adjustment. Here $z_{t}$ is located near the tropopause at $15 \mathrm{~km}$, where the specified radiative cooling vanishes. The half-sine-wave profile gives a maximum adjustment rate in the midtroposphere and might be considered to be appropriate to a first internal mode structure. Although the function $f_{2}$ tends smoothly to zero as $z$ approaches the surface; nonetheless, we calculate the WTG vertical velocity below $z_{\mathrm{BL}}$ by interpolation, as described above.

\section{b. Heat and moisture budgets}

Here we state the heat and moisture budgets for the system in order to clarify the differences between RCE, the reference-column approach, and the two-column formulation presented above. The budgets for the twocolumn formulation may be written as

$$
\sum_{i=1,2}\left\{\mathrm{SHF}_{i}+C_{p} \int_{0}^{z_{\text {top }}}\left[\left(\frac{\partial T_{i}}{\partial t}\right)_{\mu}+\left(\frac{\partial T_{i}}{\partial t}\right)_{\mathrm{rad}}+\left(\frac{\partial T_{i}}{\partial t}\right)_{\mathrm{WTG}}\right] \rho d z\right\}=0
$$

and

$$
\sum_{i=1,2}\left[E_{i}-P_{i}+L_{v} \int_{0}^{z_{\mathrm{top}}} \rho\left(\frac{\partial q_{i}}{\partial t}\right)_{\mathrm{WTG}} d z\right]=0
$$

where $C_{p}$ is the heat capacity at constant pressure and $L_{v}$ is the latent heat of vaporization. SHF is the sensible heat flux, and the three temperature tendency terms in square brackets in Eq. (12) represent the heating from phase changes, the heating from radiation, and the heating from the WTG-diagnosed circulation, respectively. In Eq. (13), $E$ and $P$ represent evaporation and precipitation, respectively.

In RCE there are no WTG contributions and the summation sign can be removed from Eqs. (12) and (13); that is, the heat and moisture budgets are balanced within each simulated column separately. In the referencecolumn approach, the summation signs should also be removed, since only the budget for a single simulated column is under consideration. The WTG contributions therefore act as external source terms. A two-column system could also have been formulated with similar source terms arising from assumed interactions with an external reservoir, in which case either $\left(\partial T_{1} / \partial t\right)_{\mathrm{WTG}} \neq$ $\left(\partial T_{2} / \partial t\right)_{\mathrm{WTG}}$ or $\left(\partial q_{1} / \partial t\right)_{\mathrm{WTG}} \neq\left(\partial q_{2} / \partial t\right)_{\mathrm{WTG}}$. However, a two-column system may also be considered as an isolated system without external source terms, as we have done here. The absence of external sources has implications for the state of the system in homogeneous conditions, as we discuss in section $4 \mathrm{~b}$. The heat and moisture budgets are satisfied only for the full, closed system rather than for each column separately. The WTG contributions are equivalent to the integral of the horizontal advection of temperature and moisture between the columns, as the integral of the vertical advection within each column is equal to zero when expressed in flux form. The use of a flux form to calculate the large-scale advective tendencies is therefore convenient in ensuring conservation of heat and moisture in the system as a whole.

\section{WTG calculations over a uniform surface}

\section{a. Reference-column approach}

In this section, we show the results from applying a reference-column approach as in Raymond and Zeng (2005), for example. In this approach one of the columns (the reference column) is held fixed. Its potential temperature and specific humidity profiles are those obtained in the RCE state of the corresponding uncoupled control run (i.e., the profiles shown in Fig. 1). The other column (the test column) is allowed to evolve and is coupled to the reference column via the WTG calculations. Thus, its domain-mean potential temperature profile is constrained to remain close to that prescribed by the reference-column state.

The RCE profiles shown in Fig. 1 are also used to initialize the test column. The WTG adjustment time scale $\tau$ is chosen to be $2 \mathrm{~h}$. An approximate indication of the associated spatial scale for the circulation is that a gravity wave moving at $50 \mathrm{~m} \mathrm{~s}^{-1}$ would travel $360 \mathrm{~km}$ in this time. The simulation is run for 40 days with the SST in the test column set equal to that of the RCE reference column, that is, $302.7 \mathrm{~K}$. Experiments with both uniform and sinusoidal WTG adjustment profiles have been used and lead to qualitatively similar results. The results presented here are those obtained from simulations performed with the uniform adjustment profile $f_{1}$.

Figure 4 shows the time series of column water vapor (CWV) (solid curve) and daily-mean rain rate (dotted curve) obtained in the test column. CWV decreases from 


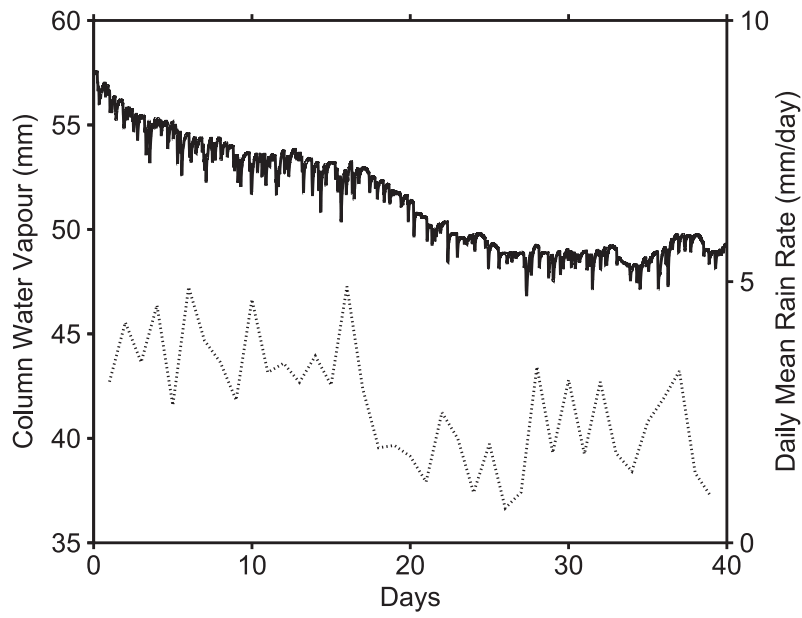

FIG. 4. Time series over 40 days of column-integrated water vapor (solid line, scale on left axis) and daily-mean rain rate (dotted line, scale on right axis) from the reference-column simulation performed with $\tau=2 \mathrm{~h}$ and the test column initialized with referencestate profiles.

its initial value equal to the RCE value, and reaches a drier quasi-steady state at around day 20 . The mean rain rate averaged between days 20 and 40 is $1.99 \mathrm{~mm}$ day $^{-1}$, much lower than that during the first 20 days of the integration and much lower than the $4.77 \mathrm{~mm}^{-1 a y}{ }^{-1}$ at RCE in the uncoupled control run. Despite the fact that the test column is initialized to have the same characteristics (thermodynamic profiles, SST, radiative cooling, domain length, etc.) as the reference column, the equilibrium state obtained by applying the WTG approximation using a reference column clearly differs from the RCE state.

The test column precipitates less than it evaporates (Fig. 5). The net imbalance of precipitation minus evaporation must be balanced by a moisture sink because of the WTG-diagnosed large-scale circulation, which corresponds to descent in the test column with convergence aloft and divergence of moist air below. The mass flux profile corresponding to the large-scale circulation is shown as the dash-dotted line in the left panel of Fig. 6. The corresponding heating and moistening source terms from the large-scale circulation are shown in the middle and right panels of Fig. 6, respectively.

\section{1) Sensitivity to WTG Adjustment time SCALE}

The sensitivity to the WTG adjustment time scale has been investigated with additional simulations using $\tau=$ $6,12,24$, and $120 \mathrm{~h}$. The mean rain rates (squares) and surface evaporation (circles) at equilibrium in each of these integrations, and in the integration with $\tau=2 \mathrm{~h}$, are plotted in Fig. 5. As $\tau$ is increased, the mean rain rate

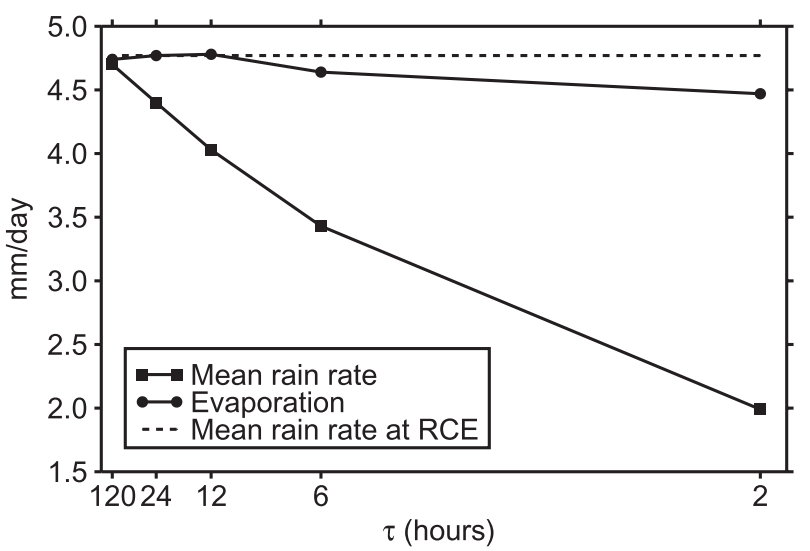

FIG. 5. Mean rain rate (squares) and mean surface evaporation (circles) at equilibrium for the simulations performed with $\tau=2,6$, 12,24 , and $120 \mathrm{~h}$, and the test column initialized with referencestate profiles. The dashed horizontal line represents the mean rain rate obtained at RCE in the uncoupled control integration. Note that the horizontal axis is scaled as the reciprocal of $\tau$.

increases toward the RCE value obtained in the uncoupled control run (indicated by the dashed horizontal line). For the simulation with $\tau=120 \mathrm{~h}$, the equilibrium evaporation and rain rates are not statistically distinguishable from the control run. For all other simulations, the test column adjusts to a new, distinct equilibrium with a lower CWV and a significantly lower mean rain rate. The equilibrium state obtained in these simulations with the test column coupled to a reference column is clearly asymmetric between the test and reference columns, a situation that is maintained by a large-scale circulation having its descending branch in the test column.

For relatively weak WTG coupling, the mean rain rate appears to reduce approximately linearly with the inverse of $\tau$, that is, with the WTG adjustment rate. For shorter coupling time scales, this linear relationship appears to break down. Surface evaporation changes are much smaller than the precipitation changes with the only significant (compared to the variability of the daily mean at RCE) reductions in surface evaporation occurring for the simulations with $\tau=2$ and $6 \mathrm{~h}$. Although there are significant variations in the equilibrium $\mathrm{CWV}$ with $\tau$, the boundary layer humidity remains relatively unchanged (not shown). The use of a mean horizontal wind (normal to the model domain) in the surface flux calculations means that the surface fluxes are relatively insensitive to the reduction in near-surface perturbation winds associated with the reduction in convective activity.

The large-scale circulation mass fluxes and the heating and the moistening rates from the large-scale circulation are shown in the left, middle, and right panels, respectively, 


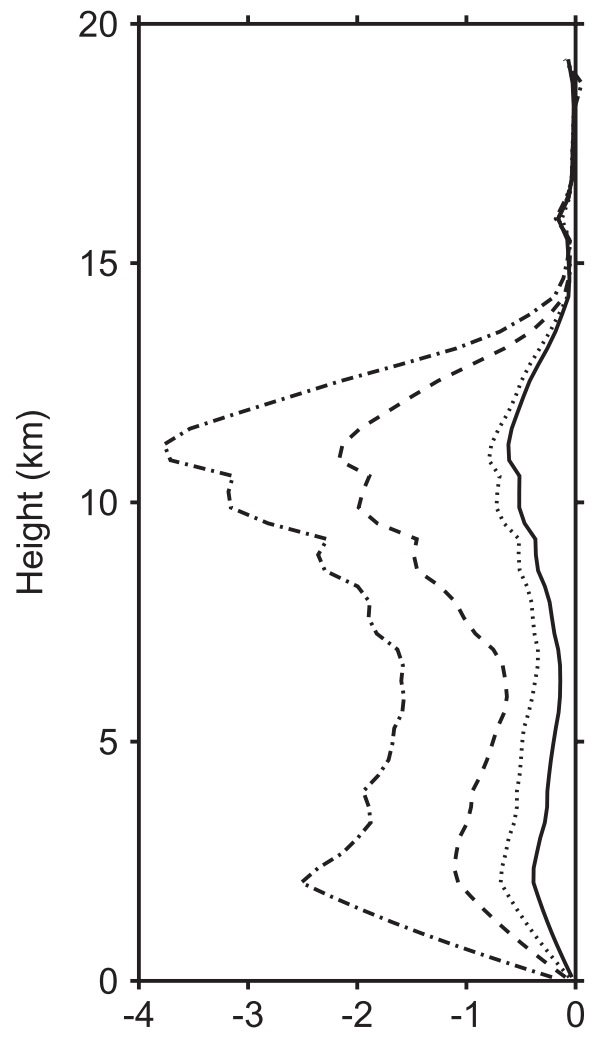

Large-scale mass fluxes $\left(\mathrm{gm}^{-2} \mathrm{~s}^{-1}\right)$

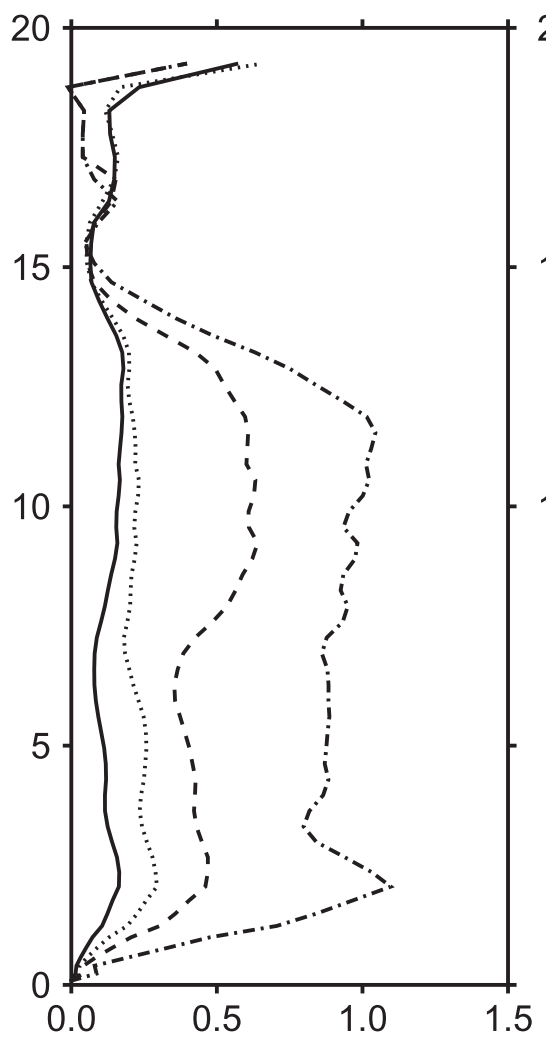

Heating

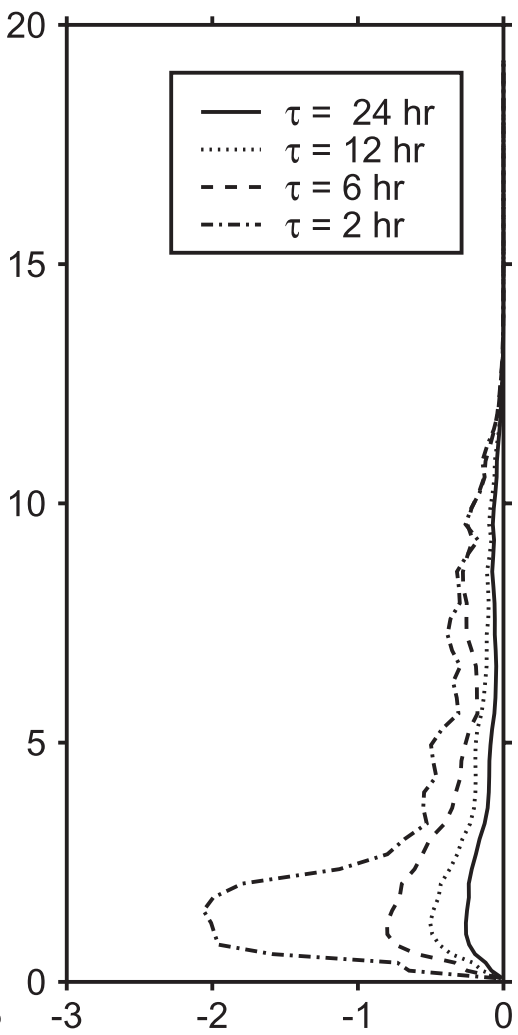

and

moistening

from the large-scale circulation ( $\mathrm{K} /$ day)

FIG. 6. (left) Large-scale mass fluxes, (middle) heating, and (right) moistening rates in the test column due to the large-scale circulation. The results are obtained by averaging the last 20 days of data from simulations performed with various values of $\tau: 2$ (dash-dotted), 6 (dashed), 12 (dotted), and $24 \mathrm{~h}$ (solid). The test column was initialized with reference-state profiles in all cases. The large-scale mass flux is computed as the product of the density and the WTG-diagnosed large-scale vertical velocity.

of Fig. 6. They correspond to subsidence warming and drying occurring within the test column, balancing the excess of evaporation over precipitation and the difference between the convective heating and the imposed radiative cooling. The large-scale mass flux profiles are large in the upper troposphere as a result of the low static stability there (Fig. 1). Note that the relatively uniform heating profile through much of the free troposphere implies a relatively uniform potential temperature difference between the test and reference columns. The drying profiles show a peak in the lower troposphere, where both the humidity and the divergence are large. The energy budget in the test column is closed by the sources due to the large-scale circulation. However, energy and moisture transported out of the test column are not received by the reference column. The significance of this point for the large-scale circulation that develops in homogeneous conditions is discussed in section $4 \mathrm{~b}$.
The presence of a large-scale circulation at equilibrium with descent in the test column is insensitive to the choice of the WTG adjustment profile. A similar circulation with the same sense has also been seen previously in similar experiments for some models (e.g., Raymond and Zeng 2005; Raymond 2007; Sessions et al. 2010), although other models do not produce a circulation under homogeneous conditions (e.g., Sobel and Bretherton 2000; Wang and Sobel 2011).

\section{2) Sensitivity to the Initial CONDitions}

To examine the sensitivity of the equilibrium state to the initial conditions, a series of simulations is performed in which the test column is initialized from RCE profiles previously obtained for other values of SST, ranging from 301.7 to $305.7 \mathrm{~K}$. The initial profiles are shown in Fig. 2 and a WTG adjustment time scale of $2 \mathrm{~h}$ is used in all cases. Figure 7 shows the time series of daily-mean rain rate from the simulations initialized 


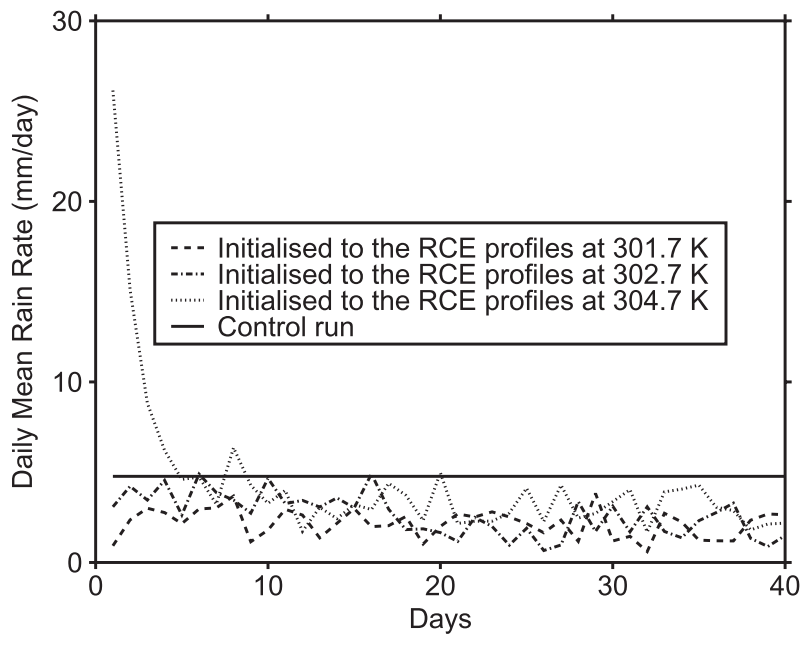

FIG. 7. Time series over 40 days of daily-mean rain rate from reference-column simulations performed with $\tau=2 \mathrm{~h}$. The test column was initialized with RCE profiles previously obtained for SSTs of 301.7 (dashed), 302.7 (dash-dotted), and $304.7 \mathrm{~K}$ (dotted). The solid horizontal line represents the mean rain rate obtained at RCE in the uncoupled control run, with SST of $302.7 \mathrm{~K}$.

with the RCE profiles at 301.7, 302.7 (i.e., the integration described in section $4 \mathrm{a}$ ), and $304.7 \mathrm{~K}$; these results provide examples of the behavior found for a larger set of simulations with the test column initialized colder than the reference profile, equal to the reference profile, and warmer than the reference profile, respectively.

Simulations in which the test column is initialized with the RCE profiles for SSTs colder than the control integration immediately develop a large-scale circulation with descent in the test column, as would be expected from the difference between the initial and reference profiles. The mean rain rate remains below that of the uncoupled control integration throughout the simulation, and the final equilibrium state is very similar to that obtained when the integration is initialized with the reference profile. Figure 8 shows the heating and moistening sources due to the large-scale circulation averaged over days $0-4$ and $30-40$ for the same integrations as in Fig. 7. The heating and moistening sources in the cold initialization case are very similar to those for the integration initialized with the reference profiles in both the early stages of the simulation and at equilibrium.

The simulations in which the test column is initialized with a profile warmer and moister than that of the reference column initially develop a large-scale circulation with ascent in the test column. Initially the rain rate exceeds that in the uncoupled control integration, but this decreases rapidly such that-by day 5 in the example shown in Fig. 7-it quickly becomes similar to that in the control integration. By day 30 a new equilibrium state has been established in which the test column precipitation is significantly lower than the control integration and resembles the integrations initialized with the referencecolumn profiles or colder.

Figure 8 shows that the large-scale ascent at early times has a top-heavy profile, partly because of the profile of the initial temperature difference (Fig. 2) and partly because of feedback from the shape of the convective heating profile that develops in response to the imposed additional cooling. In the example shown, the large-scale ascent over the first 4 days leads to a cooling that corresponds to a sink of energy from the column of $177 \mathrm{~W} \mathrm{~m}^{-2}$. To maintain ascent in the test column, sufficient moisture would have to be transported into the column so as to produce enough precipitation to balance the additional cooling. However, the energy associated with the moisture source is only $91 \mathrm{~W} \mathrm{~m}^{-2}$, as the topheavy circulation produces maximum convergence at midlevels where the humidity is relatively small. Thus, the initial large-scale circulation extracts energy from the column and the strength of the large-scale circulation reduces.

We now consider the moist static energy budget in the test column in order to investigate further how the test column evolves from an initial state with large-scale ascent to a final equilibrium state with large-scale descent. Figure 9 shows a scatterplot of the daily-mean tendency of column-integrated moist static energy in the test column due to the large-scale circulation against the difference in column-integrated moist static energy between the test and the reference columns. The plot is for the integration initialized with the warm, moist RCE profiles at $304.7 \mathrm{~K}$ described above. We also consider Fig. 10, which shows the daily-mean tendency of columnintegrated moist static energy in the test column due to the combined effects of both surface evaporation and radiation. Given that the radiative cooling is prescribed and held fixed in time, this quantity is equivalent to the difference in surface evaporation between the test column and the RCE value for the reference column. Aside from the action of the large-scale circulation on the test column, this is the only other source of difference in the column-integrated moist static energy budget between the test column and the RCE state.

The initial large-scale ascent rapidly extracts moist static energy from the test column due to the excess of cooling over moistening for the reasons discussed above. The moist static energy in the test column remains greater than that of the reference column until day 10 . From day 9, the large-scale circulation provides a source of moist static energy to the test column (Fig. 9 inset). However, the surface evaporation in the test column at this time is lower than the RCE evaporation rate appropriate for the reference-column state (Fig. 10). This 

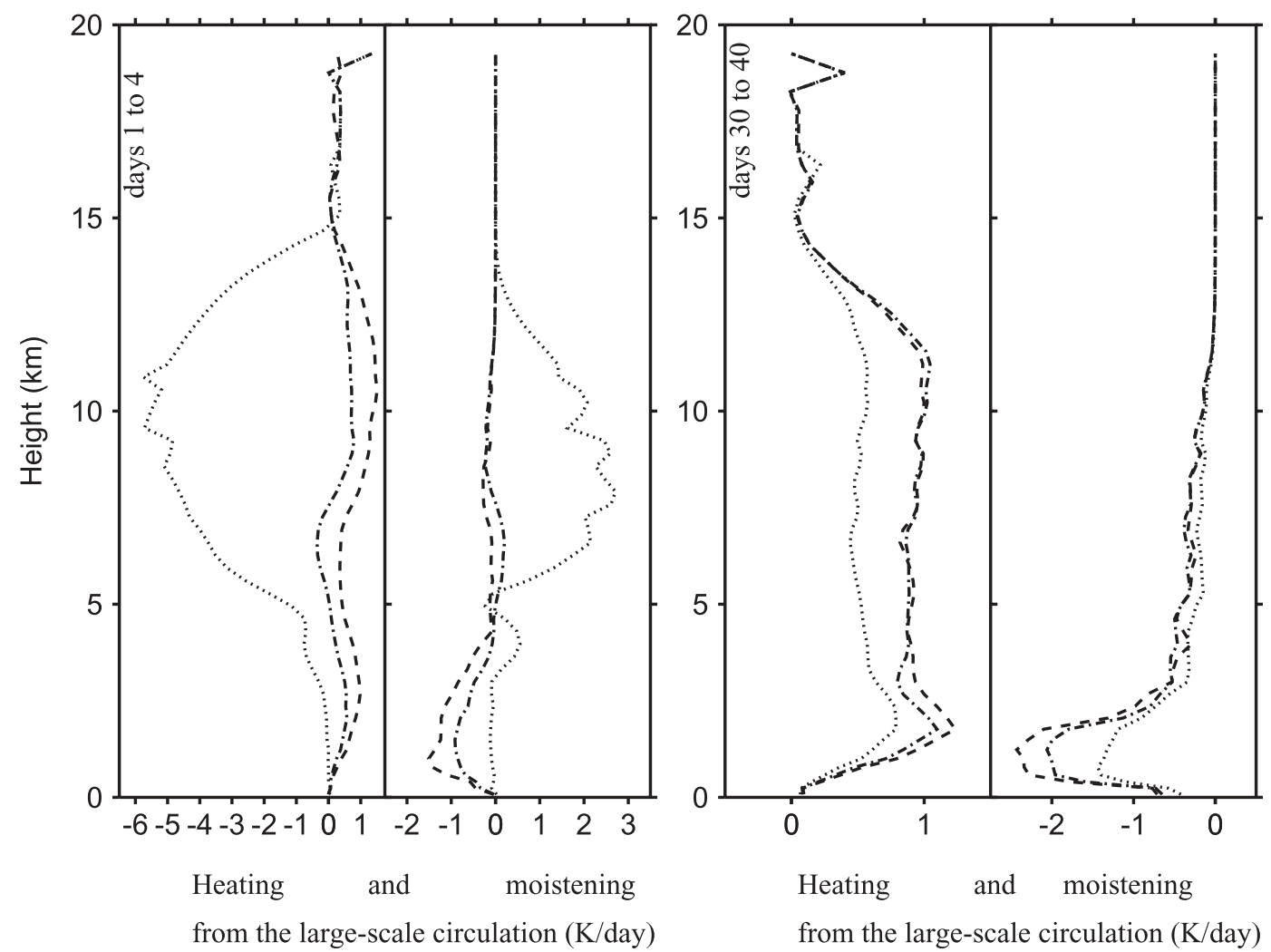

FIG. 8. Heating and moistening rates in the test column due to the large-scale circulation. The results are obtained by averaging from (left) days 0 to 4 and (right) days 30 to 40. Line definitions are as in Fig. 7.

difference is larger than the import of moist static energy into the test column and so the moist static energy continues to drop. The reduction in evaporation during this period is associated with higher boundary layer humidity in the test column compared to that in the RCE state of the reference column. At equilibrium there is a small net source of moist static energy in the test column produced by the large-scale circulation, and this is compensated by a small reduction in the surface evaporation rate compared to the uncoupled control RCE integration. Such equilibrium is established by day 30 and is very similar to the state established in the corresponding integrations initialized with the reference profile or colder.

Additional sensitivity tests were conducted with initial conditions taken from instantaneous states of RCE simulations at a range of SSTs. In all cases, the results obtained were similar, producing an equilibrium state of the coupled system with large-scale descent and reduced precipitation in the test column. Further experiments were also performed to test the sensitivity to the WTG adjustment rate profile $f(z)$, and again in all cases an equilibrium developed with descent in the test column.
In the configuration considered so far, with homogeneous surface and forcing conditions being used for the test and reference columns, an equilibrium state develops with descent in the test column. The presence of a large-scale circulation with descent in the test column under homogeneous conditions is a robust result of the simulations presented here. However, the result must be interpreted with caution and, in particular, it cannot be assumed that a large-scale circulation would develop in analogous conditions for a two-column configuration. The apparent absence of any equilibrium solution with ascent in the test column is counter intuitive to the existence of a stable equilibrium with a large-scale circulation in a configuration where the implied feedback from the test column is taken into account. In a twocolumn configuration, mass conservation implies that descent in one column must be accompanied by ascent in the other, and one might expect at least some of the simulations to be able to capture this part of a presumed full two-column equilibrium solution. As discussed in section 3b, in the reference-column approach the WTGderived circulation provides source terms to the heat and moisture budgets and in practice we have found that their evaluation produces source terms at equilibrium 


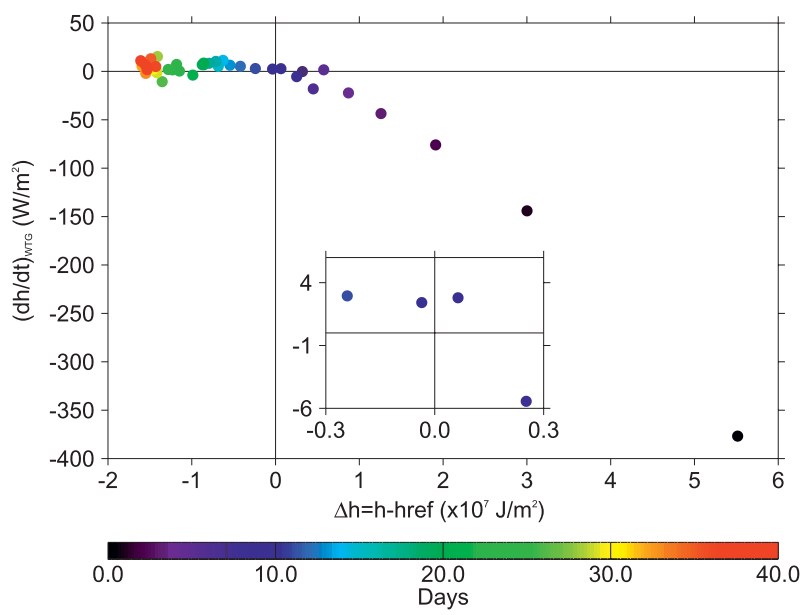

FIG. 9. Scatterplot of the difference in the column-integrated moist static energy (daily mean in the test column minus the value for the reference column) and the column-integrated moist static energy tendency due to the large-scale circulation. The results are obtained from the reference-column simulation performed with $\tau=2 \mathrm{~h}$, and the test column initialized with RCE profiles previously obtained for an SST of $304.7 \mathrm{~K}$. Each dot corresponds to a daily mean, and the color bar indicates time. The inset shows a zoom around the origin.

that always have the same sense and so are always associated with the descending branch of a large-scale circulation in the simulated column. We next test whether the existence of an equilibrium with a large-scale circulation under homogeneous forcing can be maintained in the coupled two-column system described in section 3, for which a WTG-derived circulation does not provide any net sources to heat and moisture budgets of the full system.

\section{b. Coupled-column approach}

In this section we describe results from experiments in which the WTG approximation is used to couple together two CRM simulations, with the large-scale circulation affecting both columns. Key experiments performed under the reference-column approach are repeated under the coupled-column approach. As an example, Fig. 11 shows the evolution of CWV and precipitation for the two columns, for a simulation in which column 1 is initialized to the RCE profiles at $302.7 \mathrm{~K}$, and column 2 is initialized to the RCE profiles at $304.7 \mathrm{~K}$. The SST is $302.7 \mathrm{~K}$ in both columns and $\tau=2 \mathrm{~h}$.

The CWV in column 2 gradually decreases to the same value as that in column 1 , such that by day 30 the two curves are indistinguishable and within $1 \mathrm{~mm}$ of the RCE value for the uncoupled control integration. Initially the precipitation in column 2 exceeds that in column 1 , with more than $80 \%$ of the precipitation on the first day falling in column 2 . This fraction decreases with

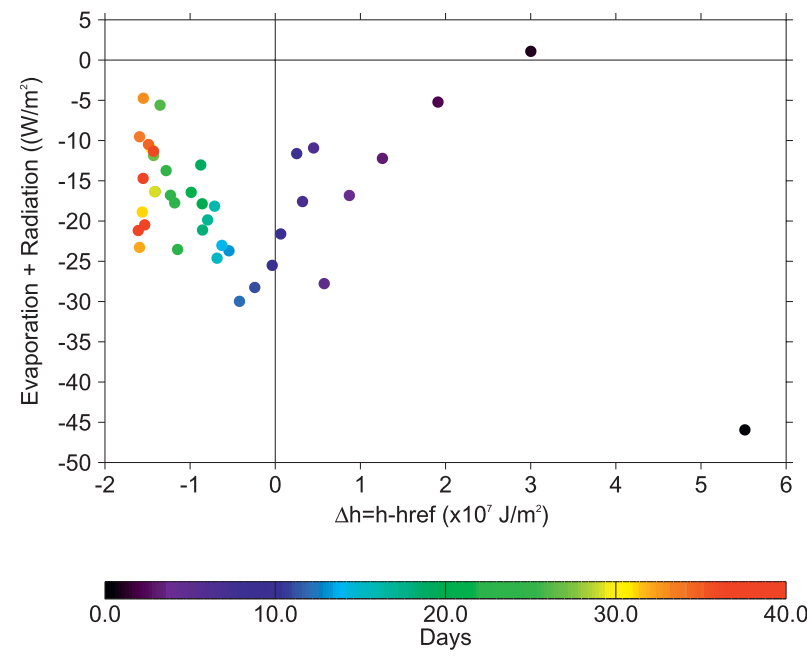

FIG. 10. As in Fig. 9, but with the $y$ axis showing the columnintegrated moist static energy tendency in the test column due to surface evaporation and radiation.

time. The precipitation averaged between days 30 and 40 is equal in the two columns to within $0.2 \mathrm{~mm} \mathrm{day}^{-1}$, and balances the imposed radiative cooling.

Figure 12 shows the daily-mean vertical velocity in column 2. Initially large-scale ascent occurs in column 2 in response to the temperature difference between the two columns. The ascent decreases in strength as the temperature difference is removed, and from about day 16 an oscillation begins to develop in the vertical velocity with periods of ascent and descent. These oscillations continue throughout the integration, but by day 30 there is no preferred column for ascent and the
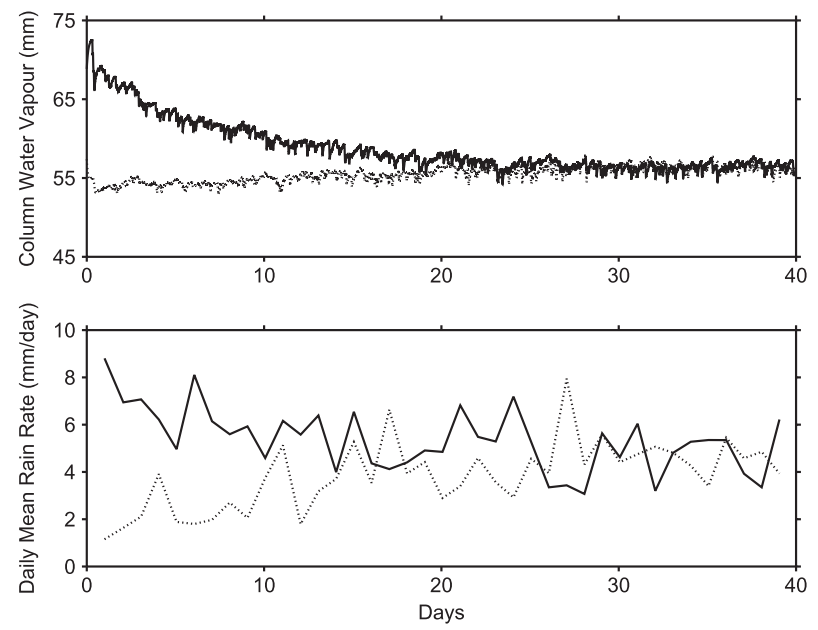

FIG. 11. Time series over 40 days of (top) CWV and (bottom) daily-mean rain rates from a coupled-column simulation performed with $\tau=2 \mathrm{~h}$. Columns 1 (dotted lines) and 2 (solid lines) are initialized to the RCE profiles previously obtained for SSTs of 302.7 and $304.7 \mathrm{~K}$, respectively. 


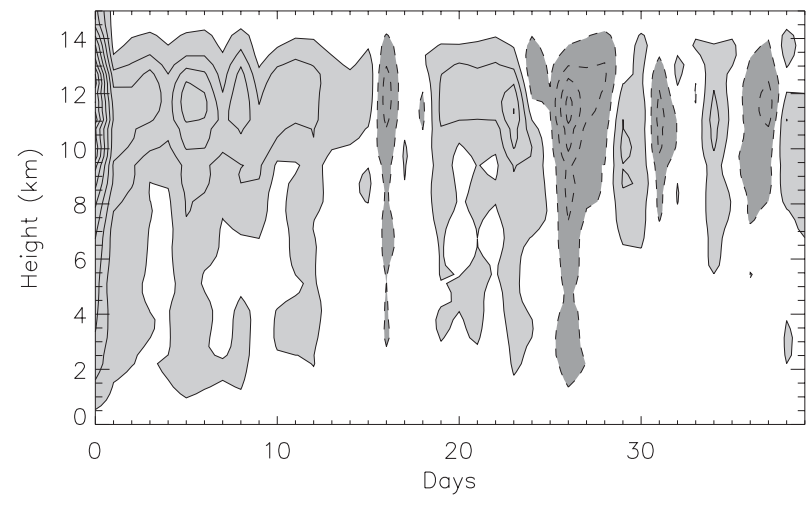

FIG. 12. Time series over 40 days of the daily-mean vertical velocity in column 2 from a coupled-column simulation performed with $\tau=2 \mathrm{~h}$. Columns 1 and 2 are initialized to the RCE profiles previously obtained for SSTs of 302.7 and $304.7 \mathrm{~K}$, respectively. The contour interval is $0.5 \mathrm{~cm} \mathrm{~s}^{-1}$, with the contours beginning at $\pm 0.25 \mathrm{~cm} \mathrm{~s}^{-1}$. Light shading and solid contours show ascent, and dark shading and dashed contours show descent.

average vertical velocity for days $30-40$ in each column is close to zero.

We now compare the behavior of this simulation to its equivalent in the reference column. Figure 13 shows the deviation from the reference profiles for the domainmean profiles at the end of day 1 of the simulations performed with $\tau=2 \mathrm{~h}$ and column 2 of the coupledcolumn model or the test column of the referencecolumn model initialized to the RCE profiles at $304.7 \mathrm{~K}$. In the coupled-column model, the initial large-scale circulation cools and moistens the warm column. At the same time, because heat and moisture are being transported, the circulation also warms and dries the cool column. The temperature difference between the columns quickly reduces, such that by the end of the first day the temperature difference above the boundary layer is small of order $0.1 \mathrm{~K}$ compared to a $0.5-2-\mathrm{K}$ temperature difference between the test column and the reference column. As a result, there is a very little circulation between the columns, and the time scale for adjustment back to the RCE profiles is governed by the moisture time scale in the system (e.g., Tompkins and Craig 1998b). In the reference-column approach, a much larger rate of precipitation can be achieved in the test column because, by construction, the cooling by the large-scale circulation in the test column is not offset by any warming of the other column. Hence, precipitation can be strong enough to dry the test column with the result that both temperature and moisture are adjusted toward RCE over the first day.

During the first 20 days, while the coupled-column system equilibrates, the mean precipitation in the columns is about $90 \%$ of that required to balance the imposed radiative cooling and the system loses heat energy.
Evaporation during the same period is about $87 \%$ of that required to balance the imposed cooling, resulting in a net loss of moist static energy and a conversion of latent energy to heat energy.

As for the reference-column approach, sensitivity experiments have been performed with different initial profiles and different values of the WTG time scale. No matter what the strength of initial temperature difference or what the strength of the initial large-scale circulation, the coupled-column system with homogeneous surface conditions was always found to adjust the columns to produce a quasi-steady state with no mean large-scale circulation and the mean statistics of the corresponding uncoupled RCE simulation.

For all of the above-mentioned simulations with the coupled-column approach, the simulated columns were of equal area (i.e., $\varepsilon=0.5$ ), a configuration for which the reference-column approach was certainly not designed; rather, the reference-column approach is intended to describe a small area embedded within a large-scale environment. As noted in section 3, a reference-column approach can be recovered from the coupled-column approach by taking the limit of $\varepsilon=0$. An interesting question arising from the above results is whether the coupled-column approach exhibits similar behavior to the reference-column approach as $\varepsilon \rightarrow 0$. We therefore repeated the experiments described in this section for homogeneous surface conditions for the cases $\varepsilon=0.1$ and $\varepsilon=0.01$. Equilibrium states with no mean largescale circulation were again established. This supports our interpretation that the character of the equilibrium state is dependent on whether the simulated system is closed, as in this section, or is open, with external source terms in the heat and moisture budgets. By construction, the coupled-column system studied here is closed for any nonzero $\varepsilon$, however small.

\section{WTG calculations for nonuniform surface conditions}

Given the marked difference in behavior between the coupled-column and reference-column approaches for homogeneous surface conditions, we now reexamine some key WTG experiments that have been reported in the literature under inhomogeneous surface conditions. Analogous experiments using the reference-column approach are repeated here and are compared with experiments using the coupled-column approach.

The sensitivity to inhomogeneous surface conditions has been investigated by changing either the SST in one of the columns [as in Sobel et al. (2007)] or the component of the wind perpendicular to the simulated domain [as in Raymond and Zeng (2005); Sessions et al. (2010)]. 

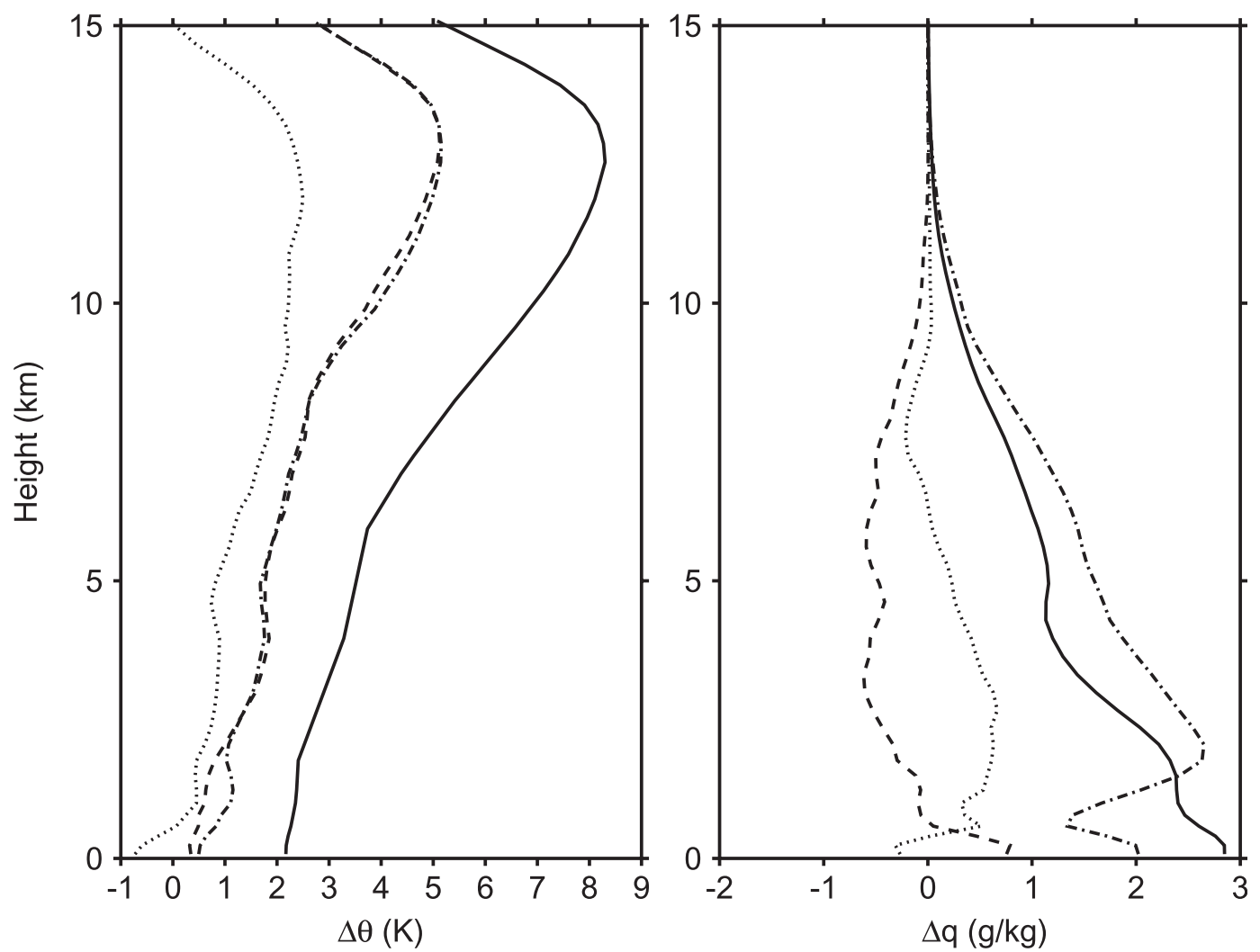

FIG. 13. Deviation from the reference profiles of (left) potential temperature and (right) specific humidity for the domain-mean profiles in the test column (dotted), in column 1 (dashed), and in column 2 (dash-dotted) at the end of day 1 of the simulations performed with $\tau=2 \mathrm{~h}$ and column 2 of the coupled-column model or the test column of the reference-column model initialized to the RCE profiles at $304.7 \mathrm{~K}$. The solid curves represent the initial potential temperature difference and the initial specific humidity difference between the columns.

We obtained broadly similar results for both methods. Results are shown for variations in wind speed in Fig. 14. For all simulations the other column (either the reference column or column 1) has an SST of $302.7 \mathrm{~K}$ and a surface wind speed of $5 \mathrm{~m} \mathrm{~s}^{-1}$.

As presented in section $4 \mathrm{a}$, the precipitation in the test column under homogeneous surface conditions is less than that in the corresponding uncoupled RCE simulation used to define the reference-column state. A mean precipitation equivalent to that of the RCE reference column is not achieved in the test column unless its surface wind speed (or SST) is increased (similar to Raymond and Zeng 2005). In the reference-column approach, there is a large sensitivity of the precipitation in the test column to the difference in surface forcing, similar in magnitude to that seen in previous studies using the reference-column approach. ${ }^{1}$ Note that for the high-surface-wind-speed simulations (specifically for

\footnotetext{
${ }^{1}$ Compare, for example, our Fig. 14 to the top panel of Fig. 11 in Raymond and Zeng (2005).
}

speeds of $10 \mathrm{~m} \mathrm{~s}^{-1}$ and above), the precipitation in the test column is more than that which would be required to balance the imposed forcing in the whole of a twocolumn system with columns of equal area. This highlights the magnitude of the WTG source term in the energy budget of the reference-column system.

Results are shown for the coupled-column approach with two different values of $\varepsilon$, specifically 0.5 (top panel) and 0.1 (bottom panel). For the case of $\varepsilon=0.1$, we doubled the value of the WTG time scale from 2 to $4 \mathrm{~h}$ in order to produce a very similar strength of the adjustment rate (the scaling of adjustment rates with area was discussed in section 3 ). In practice, other experiments altering the adjustment rate used in the coupled-column approach showed that this has little effect on the results presented in Fig. 14. As discussed in section 4b, no mean large-scale circulation develops in the coupled-column system under homogeneous surface conditions. With nonuniform surface forcing, the increase in precipitation in the column that is more strongly forced at the surface is balanced by a reduction in precipitation in the other column, such that the energy balance of the system is maintained without 

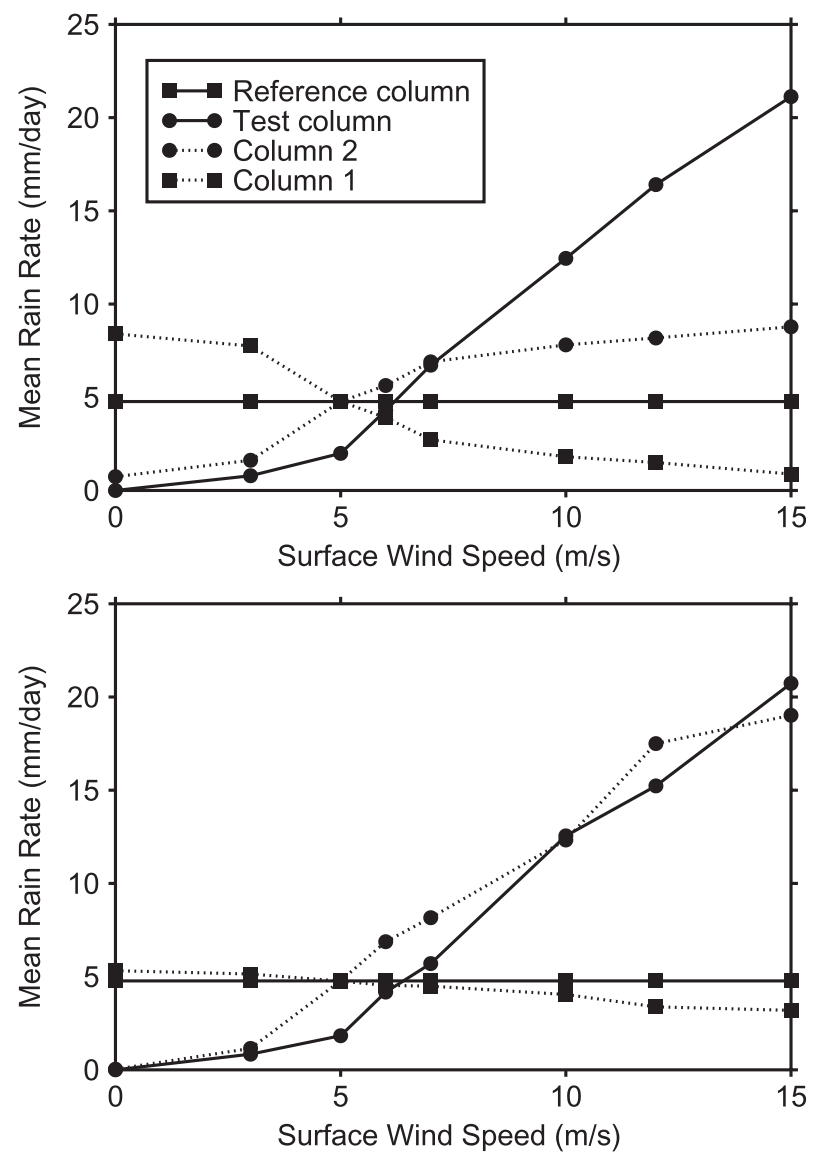

FIG. 14. Rain rates at equilibrium as a function of the surface wind speed perpendicular to the simulated domain: (top) $\varepsilon=0.5$ and $\tau=$ $2 \mathrm{~h}$ and (bottom) $\varepsilon=0.1$ and $\tau=4 \mathrm{~h}$. The results are obtained by averaging over days 25-40. Results are shown for columns 1 (dotted line with squares) and 2 (dotted line with circles) in a set of simulations performed under the coupled-column approach. The surface wind speed in column 2 is plotted on the horizontal axis. Results are also shown for the test column (solid line with circles) and the RCE reference column (solid line with squares) in a set of simulations performed under the reference-column approach. The surface wind speed in the test column is plotted on the horizontal axis.

any external source. To respect this constraint, for the case of equal column areas, the simulated precipitation is required to be much less sensitive to differences in surface forcing than in the reference-column approach. By choosing the $\varepsilon$ parameter of the coupled-column system to be small, we recover a very similar sensitivity to the difference in surface conditions as in the reference-column approach. Differences in sensitivity do, however, remain for small changes in the surface conditions.

\section{Conclusions}

The WTG approximation has been used in various studies to derive a large-scale circulation in order to couple a single-column model or cloud-resolving model to a predefined reference column (e.g., Sobel and Bretherton 2000; Raymond and Zeng 2005; Sessions et al. 2010; Sobel et al. 2007). The reference-column approach was designed to study the behavior of convection within a small area where conditions are different from the mean conditions within a much larger area to which it is coupled. Here we extend the modeling framework in order to couple two cloud-resolving model simulations. The coupled-column approach is identical to the reference-column approach in the limit of an infinite separation of the column areas, $\varepsilon=0$, but also allows for studies of WTG couplings between columns of comparable area. Two SCMs have been coupled through the WTG or similar approaches in the past (e.g., Nilsson and Emanuel 1999; Raymond and Zeng 2000; Shaevitz and Sobel 2004); however, to the best of our knowledge, this is the first time that the WTG approximation has been used to couple CRMs.

When coupled to a reference-column profile generated from a previous uncoupled RCE simulation, the coupled CRM simulation with the same forcing produced an equilibrium state characterized by descent in the test column and mean precipitation less than the RCE simulation. A similar equilibrium state was produced in Raymond and Zeng (2005), for example, although other studies indicate that this is a modeldependent result (e.g., Sobel et al. 2007; Wang and Sobel 2011). In the present study, the existence of the dry equilibrium state with suppressed convection was found to be insensitive to the choice of the WTG adjustment or to the initial conditions in the test column, although the time scale for the adjustment to equilibrium and the strength of the circulation established are sensitive to the WTG time scale.

The equilibrium state found here is associated with the net transport of moist static energy into the test column by the WTG circulation with a small compensating reduction in the surface evaporation. The inability of the reference-column approach to produce an equilibrium state with ascent in the test column is related to the shape of the large-scale vertical velocity profile generated. For circulations with ascent in the test column, the velocity profile is top heavy with the maximum convergence above $5 \mathrm{~km}$. The result of this topheavy velocity profile is that the moisture convergence from the circulation is not sufficient to balance the cooling and so moist static energy is lost from the test column. As the simulation approaches the near-radiative equilibrium conditions, a weak import of moist static energy into the column by the large-scale circulation is observed but this is not sufficient to balance a reduction in the surface fluxes associated with high boundary layer 
humidity. Thus, the moist static energy of the test column is reduced further until a new equilibrium is reached with descent in the test column.

The presence of an equilibrium with descent in the test column, and the absence of an equilibrium solution with ascent in the test column, is somewhat counterintuitive. Despite the homogeneous forcing that is applied in these experiments, the test column and the reference column are not interchangeable. Moreover, the energy budget of the entire system is not closed in the referencecolumn approach.

To explore the role of the fixed reference column in producing this dry equilibrium state of the test column, we have developed an extension of the WTG approach to couple together two CRMs. In this fully coupled configuration, the budgets of the total system are now closed.

A number of experiments with homogeneous surface forcing were performed in the coupled-column approach in order to compare its behavior to the reference-column approach. In all cases, the coupled-column system reaches an equilibrium that has no time-mean large-scale circulation between the two columns and is very similar to the equilibrium state of a single uncoupled CRM. In particular, this equilibrium was insensitive to the magnitude of the initial temperature difference between the two columns, to the WTG adjustment time scale and profile, and to the relative areas of the two columns. As suggested by the contrasting results of previous studies, it is possible that the presence, or otherwise, of a dry equilibrium state in the reference-column approach may depend on the choice of the representation of convection or boundary layer mixing, or on some details of the implementation of the WTG framework. However, the key finding here is that even for small $\varepsilon$, the fully coupled approach produces a qualitatively different equilibrium state from the reference-column approach.

Most of the WTG studies to date have followed a reference-column approach and have focused on the sensitivity of the simulated precipitation in the test column to the surface forcing. We performed a similar set of experiments with both the reference-column approach and the coupled-column approach. For columns with very different areas, the coupled-column system behaves very similarly to the analogous configuration using the reference-column approach, which for our model has a similar sensitivity to previous studies. For columns of equal area, the coupled-column approach results in much weaker sensitivity to surface forcing than the referencecolumn approach. This can be understood as a consequence of the strong constraint on the extent of possible sensitivity in the coupled-column approach, due to the prescribed cooling in combination with the closed energy budget of that system. This combination sets the total precipitation in the two columns in the coupled-column approach. No such constraint applies in the referencecolumn approach, even for conditions of prescribed cooling.

The differences in behavior of the coupled-column and reference-column approaches highlight the importance of careful consideration of the appropriate energy and moisture budgets when interpreting results from studies of this nature. Future studies with the coupledcolumn approach are planned to investigate various aspects of the interaction between large-scale dynamics and convection, including the role of interactive radiation, variability in convection, and transitions between shallow and deep convection.

Acknowledgments. We thank the Met Office for the availability of the LEM, version 2.4. We are grateful for the comments provided by Adam Sobel and two anonymous reviewers. CLD was supported by the University of Reading Postgraduate Studentship (International). SJW was supported by the National Centre for Atmospheric Science, a NERC collaborative center.

\section{REFERENCES}

Abel, S. J., and B. J. Shipway, 2007: A comparison of cloudresolving model simulations of trade wind cumulus with aircraft observations taken during RICO. Quart. J. Roy. Meteor. Soc., 133, 781-794.

Bellon, G., and A. H. Sobel, 2010: Multiple equilibria of the Hadley circulation in an intermediate-complexity axisymmetric model. J. Climate, 23, 1760-1778.

Bretherton, C. S., and P. K. Smolarkiewcz, 1989: Gravity waves, compensating subsidence and detrainment around cumulus clouds. J. Atmos. Sci., 46, 740-759.

Brown, P., and A. Heymsfield, 2001: The microphysical properties of tropical convective anvil cirrus: A comparison of model and observations. Quart. J. Roy. Meteor. Soc., 127, 1535-1550.

Cohen, B. G., and G. C. Craig, 2006: Fluctuations in an equilibrium convective ensemble. Part II: Numerical experiments. J. Atmos. Sci., 63, 2005-2015.

Dai, A., 2006: Precipitation characteristics in eighteen coupled climate models. J. Climate, 19, 4605-4630.

Garner, S. T., D. M. W. Frierson, I. M. Held, O. Paulius, and G. K. Vallis, 2007: Resolving convection in a global hypohydrostatic model. J. Atmos. Sci., 64, 2061-2075.

Grabowski, W. W., J.-I. Yano, and M. W. Moncrieff, 2000: Cloud resolving modeling of tropical circulations driven by largescale SST gradients. J. Atmos. Sci., 57, 2022-2039.

Held, I. M., R. S. Hemler, and V. Ramaswamy, 1993: Radiativeconvective equilibrium with explicit two-dimensional moist convection. J. Atmos. Sci., 50, 3909-3927.

Holloway, C. E., and J. D. Neelin, 2010: Temporal relations of column water vapor and tropical precipitation. J. Atmos. Sci., 67, 1091-1105.

- S. J. Woolnough, and G. M. S. Lister, 2012: Precipitation distributions for explicit versus parameterized convection in a large-domain high-resolution tropical case study. Quart. J. Roy. Meteor. Soc., doi:10.1002/qj.1903, in press. 
Kuang, Z., 2008: Modeling the interaction between cumulus convection and linear gravity waves using a limited-domain cloud system-resolving model. J. Atmos. Sci., 65, 576-591.

_ 2011: The wavelength dependence of the gross moist stability and the scale selection in the instability of column-integrated moist static energy. J. Atmos. Sci., 68, 61-74.

- P. N. Blossey, and C. S. Bretherton, 2005: A new approach for 3D cloud-resolving simulations of large-scale atmospheric circulation. Geophys. Res. Lett., 32, L02809, doi:10.1029/ 2004 GL021024.

Lin, J.-L., and Coauthors, 2006: Tropical intraseasonal variability in 14 IPCC AR4 climate models. Part I: Convective signals. J. Climate, 19, 2665-2690.

—, M.-I. Lee, D. Kim, I.-S. Kang, and D. M. W. Frierson, 2008: The impacts of convective parameterization and moisture triggering on AGCM-simulated convectively coupled equatorial waves. J. Climate, 21, 883-909.

Liu, P., and Coauthors, 2009: An MJO simulated by the NICAM at 14- and 7-km resolutions. Mon. Wea. Rev., 137, 3254-3268.

Mapes, B. E., 1997: Equilibrium vs. activation control of large-scale variations of tropical deep convection. The Physics and Parameterization of Moist Atmospheric Convection, R. K. Smith, Ed., Kluwer Academic Publishers, 321-358.

_ , and R. A. Houze Jr., 1995: Diabatic divergence profiles in western Pacific mesoscale convective systems. J. Atmos. Sci., 52, 1807-1828.

_ and X. Wu, 2001: Convective eddy momentum tendencies in long cloud-resolving model simulations. J. Atmos. Sci., 58, 517-526.

Masunaga, H., 2012: A satellite study of the atmospheric forcing and response to moist convection over tropical and subtropical oceans. J. Atmos. Sci., 69, 150-167.

Neelin, J. D., and N. Zeng, 2000: A quasi-equilibrium tropical circulation model-Formulation. J. Atmos. Sci., 57, 17411766.

Nilsson, J., and K. A. Emanuel, 1999: Equilbrium atmospheres of a two-column radiative-convective model. Quart. J. Roy. Meteor. Soc., 125, 2239-2264.

Petch, J. C., and M. E. B. Gray, 2001: Sensitivity studies using a cloud-resolving model simulation of the tropical west Pacific. Quart. J. Roy. Meteor. Soc., 127, 2287-2306.

_ A. R. Brown, and M. E. B. Gray, 2006: The impact of horizontal resolution on the simulations of convective development over land. Quart. J. Roy. Meteor. Soc., 132, 2031-2044.

Randall, D. A., M. Khairoutdinov, A. Arakawa, and W. Grabowski, 2003: Breaking the cloud parameterization deadlock. Bull. Amer. Meteor. Soc., 84, 1547-1564.

_ Climate Change 2007: The Physical Basis, S. Solomon et al., Eds., Cambridge University Press, 589-662.

Raymond, D. J., 2007: Testing a cumulus parametrization with a cumulus ensemble model in weak-temperature-gradient mode. Quart. J. Roy. Meteor. Soc., 133, 1073-1085.

— , and X. Zeng, 2000: Instability and large-scale circulations in a two-column model of the tropical troposphere. Quart. J. Roy. Meteor. Soc., 126, 3117-3136.

—_, and —-, 2005: Modelling tropical atmospheric convection in the context of the weak temperature gradient approximation. Quart. J. Roy. Meteor. Soc., 131, 1301-1320.

Robe, F. R., and K. A. Emanuel, 1996: Moist convective scaling: Some inferences from three-dimensional cloud ensemble simulations. J. Atmos. Sci., 53, 3265-3275.
— and - 2001: The effect of vertical wind shear on radiativeconvective equilibrium states. J. Atmos. Sci., 58, 1427-1445.

Rotunno, R., J. Klemp, and M. Weisman, 1988: A theory for strong, long-lived squall lines. J. Atmos. Sci., 45, 463-485.

Sessions, S. L., S. Sugaya, D. J. Raymond, and A. H. Sobel, 2010: Multiple equilibria in a cloud-resolving model using the weak temperature gradient approximation. J. Geophys. Res., 115, D12110, doi:10.1029/2009JD013376.

Shaevitz, D., and A. Sobel, 2004: Implementing the weak temperature gradient approximation with a full vertical structure. Mon. Wea. Rev., 132, 662-669.

Shutts, G. J., and M. E. B. Gray, 1994: A numerical modelling study of the geostrophic adjustment process following deep convection. Quart. J. Roy. Meteor. Soc., 120, 1145-1178.

— , and T. N. Palmer, 2007: Convective forcing fluctuations in a cloud-resolving model: Relevance to the stochastic parameterization problem. J. Climate, 20, 187-202.

Sobel, A. H., and C. S. Bretherton, 2000: Modeling tropical precipitation in a single column. J. Climate, 13, 4378-4392.

— intertropical convergence zones in the quasi-equilibrium tropical circulation model framework. Theor. Comput. Fluid Dyn., 20, 323-350.

, G. Bellon, and J. Bacmeister, 2007: Multiple equilibria in a single-column model of the tropical atmosphere. Geophys. Res. Lett., 34, L22804, doi:10.1029/2007GL031320.

Swann, H., 1998: Sensitivity to the representation of precipitating ice in CRM simulations of deep convection. Atmos. Res., 4748, 415-435.

Tao, W., J. Simpson, C. Sui, C. Shie, B. Zhou, K. Lau, and M. Moncrieff, 1999: Equilibrium states simulated by cloudresolving models. J. Atmos. Sci., 56, 3128-3139.

Tompkins, A. M., 2000: The impact of dimensionality on long-term cloud-resolving model simulations. Mon. Wea. Rev., 128, 1521-1535.

_ 2001: Organization of tropical convection in low vertical wind shears: The role of water vapor. J. Atmos. Sci., 58, 529-545.

—, and G. C. Craig, 1998a: Radiative-convective equilibrium in a three-dimensional cloud-ensemble model. Quart. J. Roy. Meteor. Soc., 124, 2073-2097.

$\longrightarrow$, and — 1998b: Time-scales of adjustment to radiativeconvective equilibrium in the tropical atmosphere. Quart. J. Roy. Meteor. Soc., 124, 2693-2713.

Vincent, D., 1994: The South Pacific convergence zone (SPCZ): A review. Mon. Wea. Rev., 122, 1949-1970.

Wang, S., and A. H. Sobel, 2011: Response of convection to relative sea surface temperature: Cloud-resolving simulations in two and three dimensions. J. Geophys. Res., 116, D11119, doi:10.1029/2010JD015347.

Webster, P. J., and R. Lukas, 1992: TOGA COARE: The Coupled Ocean-Atmosphere Response Experiment. Bull. Amer. Meteor. Soc., 73, 1377-1416.

Woolnough, S., and Coauthors, 2010: Modelling convective processes during the suppressed phase of a Madden-Julian oscillation: Comparing single-column models with cloudresolving models. Quart. J. Roy. Meteor. Soc., 136, 333-353.

$\mathrm{Xu}, \mathrm{K} .-\mathrm{M}$., and Coauthors, 2002: An intercomparison of cloudresolving models with the Atmospheric Radiation Measurement summer 1997 intensive observation period data. Quart. J. Roy. Meteor. Soc., 128, 593-624.

Yano, J.-I., and M. Bonazzola, 2009: Scale analysis for the large-scale tropical atmospheric dynamics. J. Atmos. Sci., 66, 159-172. 